\title{
PPAR $\beta / \delta$ accelerates bone regeneration in diabetic mellitus by enhancing AMPK/mTOR pathway-mediated autophagy
}

Miao Chen ${ }^{1,2}$, Dian Jing ${ }^{3}$, Rui Ye $e^{1,2}$, Jianru Yi ${ }^{1,2^{*}}$ and Zhihe Zhao ${ }^{1,2^{*}}$

\begin{abstract}
Background: Diabetic patients are more vulnerable to skeletal complications. Peroxisome proliferators-activated receptor (PPAR) $\beta / \delta$ has a positive regulatory effect on bone turnover under physiologic glucose concentration; however, the regulatory effect in diabetes mellitus has not been investigated yet. Herein, we explored the effects of PPAR $\beta / \delta$ agonist on the regeneration of diabetic bone defects and the osteogenic differentiation of rat bone marrow mesenchymal stem cells (rBMSCs) under a pathological high-glucose condition.

Methods: We detected the effect of PPAR $/ \delta$ agonist on osteogenic differentiation of rBMSCs in vitro and investigated the bone healing process in diabetic rats after PPAR $\beta / \delta$ agonist treatment in vivo. RNA sequencing was performed to detect the differentially expressed genes and enriched pathways. Western blot was performed to detect the autophagy-related protein level. Laser confocal microscope (LSCM) and transmission electron microscope (TEM) were used to observe the formation of autophagosomes.
\end{abstract}

Results: Our results demonstrated that the activation of PPAR $\beta / \delta$ can improve the osteogenic differentiation of rBMSCs in high-glucose condition and promote the bone regeneration of calvarial defects in diabetic rats, while the inhibition of PPAR $\beta / \delta$ alleviated the osteogenic differentiation of rBMSCs. Mechanistically, the activation of PPAR $/ \delta$ up-regulates AMPK phosphorylation, yielding mTOR suppression and resulting in enhanced autophagy activity, which further promotes the osteogenic differentiation of rBMSCs in high-glucose condition. The addition of AMPK inhibitor Compound C or autophagy inhibitor 3-MA inhibited the osteogenesis of rBMSCs in high-glucose condition, suggesting that PPAR $\beta / \delta$ agonist promotes osteogenic differentiation of rBMSCs through AMPK/mTOR-regulated autophagy.

Conclusion: In conclusion, our study demonstrates the potential role of PPAR $\beta / \delta$ as a molecular target for the treatment of impaired bone quality and delayed bone healing in diabetic patients for the first time.

Keywords: PPARß/ $\delta$, Diabetes mellitus, Autophagy, Osteogenic differentiation, Bone regeneration, AMPK/mTOR pathway

\footnotetext{
*Correspondence: jianruyi@scu.edu.cn; zhzhao@scu.edu.cn

${ }^{1}$ State Key Laboratory of Oral Diseases and National Clinical Research

Center for Oral Diseases, West China Hospital of Stomatology, Sichuan University, Chengdu, China

Full list of author information is available at the end of the article
}

\begin{abstract}
Background
Diabetes mellitus is a metabolic disease characterized by hyperglycemia that can lead to chronic damage to various organs and tissues, further causing profound consequences on patients' quality of life. Due to the incessantly increasing incidence in the past decades, diabetes mellitus has become a global public problem that endangers human health [1]. According to the World Health Organization's report on diabetes mellitus, the number of
\end{abstract} original author(s) and the source, provide a link to the Creative Commons licence, and indicate if changes were made. The images or other third party material in this article are included in the article's Creative Commons licence, unless indicated otherwise in a credit line to the material. If material is not included in the article's Creative Commons licence and your intended use is not permitted by statutory regulation or exceeds the permitted use, you will need to obtain permission directly from the copyright holder. To view a copy of this licence, visit http://creativecommons.org/licenses/by/4.0/. The Creative Commons Public Domain Dedication waiver (http://creativeco mmons.org/publicdomain/zero/1.0/) applies to the data made available in this article, unless otherwise stated in a credit line to the data. 
adults living with diabetes worldwide was 422 million in 2014, which was estimated to rise to 550 million by 2030 $[2,3]$. The occurrence of diabetes mellitus has increased rapidly in developing countries during the past 10 years along with the urbanization process and changes in lifestyle. Currently, China has become the country with the most diabetic patients worldwide[4].

Diabetic patients are more vulnerable to skeletal complications, which are called as "diabetic bone disease" or "diabetic osteopathy" [5]. The common diabetic bone diseases include osteoporosis, increased fracture risk and poor bone healing properties [6]. Numerous studies demonstrated that the fracture risk in diabetic patients is higher than that in healthy individuals [7-9]. In addition, the healing time of fracture in diabetic patients would be greatly prolonged $[10,11]$. The molecular modulation of bone metabolism in diabetic environment has also been extensively explored. The hyperglycemia condition inhibits the maturation and metabolism of mesenchymal stem cells (MSCs) [12, 13]. The pathologically high-glucose environment has been proven to promote osteoblast apoptosis and inhibit osteogenic differentiation. In diabetic patients, the increased osteoblast apoptosis led to decreased bone remodeling activity $[14,15]$. The aforementioned pathological mechanisms ultimately result in bone loss and impaired bone quality. Effective therapeutic methods to promote the bone regeneration in hyperglycemia environment are essential to improve the life quality of patients with diabetic bone diseases.

Peroxisome proliferators-activated receptor (PPAR) $\beta / \delta$ is a type of ligand-activated receptor, belonging to nuclear-receptor superfamily. When activated by endogenous ligands or artificial agonists, PPAR $\beta / \delta$ acts as a transcription factor through binding to the peroxisome proliferator response elements (PPREs) located in the promoter region of targets genes [16]. PPAR $\beta / \delta$ controls a multitude of metabolic processes including lipid catabolism, glucose homeostasis, redox balance, inflammation and differentiation [17, 18]. Additionally, PPAR $\beta / \delta$ has therapeutic potential to promote the mammalian regeneration of bone, skin, muscle and liver [16]. The regulatory effect of PPAR $\beta / \delta$ on bone turnover was firstly recognized in 2013 [19]. Conditional knockout of PPAR $\beta / \delta$ in mice via Runx2-cre led to the energy metabolism disorder in osteoblasts, thus impaired the bone mineralization and bone mass [20]. Absence of $\operatorname{PPAR} \beta / \delta$ led to glucose intolerance and impaired bone formation [21]. These studies provide a new mechanism to explain the increased fracture risk in diabetes and consider PPAR $\beta / \delta$ as a potential molecular target for the treatment of bone fragility in diabetes. In addition, the activation of PPAR $\beta / \delta$ accelerated osteoblast differentiation and increased the number of peroxisomes, which were ubiquitous organelles in eukaryotic cells to regulate ROS levels [22]. Furthermore, PPAR $\beta / \delta$ has been found to restore the metabolic homeostasis through enhancing insulin sensitivity and regulating glucose metabolism in diabetic mice $[23,24]$. Taken together, PPAR $\beta / \delta$ seems to be a potential therapeutic target to treat the impaired bone formation in diabetes. However, this hypothesis has not been investigated yet.

The objective of the present study was to determine the effects of PPAR $\beta / \delta$ on bone regeneration in diabetic condition. In this study, we firstly studied the osteogenic differentiation effect of PPAR $\beta / \delta$ agonist on rat bone marrow mesenchymal stem cells (rBMSCs) in hyperglycemia environment. Secondly, we established a rat calvarial defect model to evaluate the effects of PPAR $\beta / \delta$ agonist on the healing of bone defects in diabetes. Thirdly, we explored the mechanisms of PPAR $\beta / \delta$ mediated osteogenesis in hyperglycemia condition based on RNA sequencing analysis and performed further validation for the potential mechanisms.

\section{Materials and methods \\ Experimental animals}

A total of 32 Sprague-Dawley (SD) rats at an age of 7 weeks were used. These rats were housed in a pathogen-free environment with access to standard rat food and plain water ad libitum under a $12 \mathrm{~h}$ light/dark cycle. After a 1-week acclimatization, the SD rats were randomly divided into the following four groups of eight rats each: (1) diabetes mellitus group (DM); (2) diabetes mellitus treated with PPAR $\beta / \delta$ agonist GW501516 group $(\mathrm{DM}+\mathrm{GW})$; (3) control group (Control) and (4) control treated with GW501516 (Control + GW).

$\mathrm{DM}+\mathrm{GW}$ group and Control $+\mathrm{GW}$ group were injected with GW501516 (5 mg/kg/d) dissolved in $0.1 \mathrm{ml}$ dimethyl sulfoxide (DMSO) every other day [25]. DM and Control group were injected with $0.1 \mathrm{ml}$ DMSO every other day as control. The rats were sacrificed 4 and 8 weeks after surgery. The samples were harvested for histology analysis and $\mu \mathrm{CT}$ assay.

DM model was induced by intraperitoneal injection of $1 \%$ streptozotocin (STZ) solution with a dose of $60 \mathrm{mg} /$ $\mathrm{Kg}$. The STZ solution was prepared by dissolving STZ (Sigma-Aldrich) in $0.1 \mathrm{mM}$ citric acid-citrate sodium buffer (pH 4.5) [26]. The citric acid-citrate sodium buffer without STZ was intraperitoneally injected into normal rats as control. The experimental rats were fasted for $12 \mathrm{~h}$ before STZ administration. The blood glucose of rats after a 12-h fasting was monitored on day 7 and 14 after streptozotocin injection. The rats with a fasting blood sugar values higher than $16.65 \mathrm{mmol} / \mathrm{L}$ were considered eligible [26]. 
Calvarial defect model was established in all experimental rats. Each rat was anesthetized with $4 \%(\mathrm{w} / \mathrm{v})$ isoflurane, followed by an intraperitoneal injection of ketamine $(60 \mathrm{mg} / \mathrm{kg}$, Sigma-Aldrich) and xylazine (12 mg/ $\mathrm{kg}$, Sigma-Aldrich) [27]. Before the incision was made, bupivacaine $(0.25 \%, 1-2 \mathrm{mg} / \mathrm{kg}$ dose) was used to relieve local pain. The surgical area was shaved, and a $1 \mathrm{~cm}$ midline skin incision was made between the rats' ears. Periosteum were separated to fully expose the calvarial bone. The bone defect areas with a diameter of $3 \mathrm{~mm}$ on both sides of cranial suture were prepared by dental trephine under normal saline rinsing [28]. The periosteum and skin incisions were sutured in layers with absorbable sutures after surgery.

\section{Micro-computed tomography $(\mu \mathrm{CT})$ and histomorphometrical analyses}

Calvarial bone were dissected and fixed in $4 \%$ paraformaldehyde for 2 days and then stored in $70 \%$ ethanol at $4{ }^{\circ} \mathrm{C}$. The $\mu \mathrm{CT}$ analysis was performed $(\mu \mathrm{CT} 50$, SCANCO Medical) with a spatial resolution of $10 \mu \mathrm{m}$ (55 kV, $114 \mathrm{~mA}, 500 \mathrm{~ms}$ integration time). The region of interest (ROI) was defined as a cylindrical area covering the initial bone defect with a diameter of $3 \mathrm{~mm}$ to analyze the regeneration of cortical bone [29]. Bone mineral density (BMD) and bone volume/total volume (BV/TV) were evaluated within the delimited ROI [30].

\section{Histology staining}

Following $\mu \mathrm{CT}$ analysis, the samples were decalcified with $10 \%$ EDTA for 4 weeks. Sections of $4.5 \mu \mathrm{m}$ were prepared using a conventional method [31]. H\&E and Masson trichrome staining (Solarbio, Beijing, China) were performed as the manufacturer's instruction. The stained sections were observed using an inverted microscope (IX81, Olympus).

\section{Cell culture}

The rBMSCs were obtained from Cyagen Biosciences. The femur and tibia of sacrificed 4-week-old rats were separated after soaking in $75 \%$ ethanol for $5 \mathrm{~min}$. The two ends of epiphysis were cut off to flush out the bone marrow, and the suspension was centrifuged. The supernatant was discarded, and the cells were transferred to DMEM (Gibco, Grand Island, NY, USA) with $5.6 \mathrm{mM}$ glucose concentration containing $10 \%$ fetal bovine serum (Hyclone Laboratories, Logan, UT, USA), $2 \mathrm{mM}$ L-glutamine (Sigma), $100 \mathrm{U} / \mathrm{mL}$ penicillin and $100 \mathrm{mg} / \mathrm{mL}$ streptomycin (Hyclone) at $37{ }^{\circ} \mathrm{C}$ in $5 \% \mathrm{CO}_{2}$. The medium was changed every 3 days to discard non-adherent cells [32]. The cells were expanded when the confluency reached approximately $80 \%$. The cells from passage 2 to passage 4 were used in this study.
To obtain the bone marrow-derived macrophages (BMDMs), the bone marrow was flashed into a petri dish and centrifuged as previous described. The supernatant was discarded, and the red blood cell lysate was added. The cells were seeded in a $10-\mathrm{cm}$-diameter petri dish, cultured in DMEM medium with $10 \%$ fetal bovine serum (FBS), and supplied with $50 \mathrm{ng} / \mathrm{ml} \mathrm{m}$-CSF (R\&D). After 4 days, the suspension cells were discarded and BMDMs were obtained for subsequent experiment.

For osteogenic differentiation, rBMSCs were induced with medium containing $10 \mathrm{mM} \beta$-glycerophosphate, $10^{-8} \mathrm{M}$ dexamethasone and $50 \mu \mathrm{g} / \mathrm{ml} \mathrm{L}$-2-ascorbic acid (Sigma-Aldrich, St. Louis, MO, USA) [33]. The culture cells were randomized into normal-glucose (NG) group $(5.6 \mathrm{mM})$ and high-glucose (HG) group $(30 \mathrm{mM})$. The HG medium was obtained by adding D-glucose (SigmaAldrich) to the NG medium. The NG and HG group were then divided into three subgroups and added with $0 \mu \mathrm{M}$, $1 \mu \mathrm{M}$, or $5 \mu \mathrm{M}$ of PPAR $\beta / \delta$ agonist GW501516 (SigmaAldrich), respectively, yielding a total of six groups: N0 (5.6 mM glucose), N1 (5.6 mM glucose $+1 \mu \mathrm{M}$ GW501516), N5 (5.6 mM glucose +5 $\mu \mathrm{M}$ GW501516), H0 (30 mM glucose), H1 (30 $\mathrm{mM}$ glucose $+1 \mu \mathrm{M}$ GW501516), and H5 (30 $\mathrm{mM}$ glucose $+5 \quad \mu \mathrm{M}$ GW501516).

\section{Alkaline phosphatase staining}

After 7 days of osteogenic induction, alkaline phosphatase (ALP) staining was performed using a BCIP/ NBT ALP color development kit (Beyotime, C3206, China) following manufacturer's instruction. Briefly, after removing the culture medium, the cells were washed three times with PBS and fixed with $4 \%$ polyoxymethylene for $15 \mathrm{~min}$. Then, the cells were immersed by BCIP/ NBT staining working solution and incubated at room temperature for $30 \mathrm{~min}$ in dark. The color reaction was terminated by distilled water. ALP quantification was performed with an alkaline phosphatase assay kit (Beyotime, P0321M, China) according to the manufacturer's protocol. The optical density (OD) was evaluated by measuring the absorbance at $405 \mathrm{~nm}$ spectrophotometrically. The total protein content was measured at $\mathrm{OD}_{562 \mathrm{~nm}}$, and the concentration was calculated according to the standard BSA curve using the enhanced BCA protein assay (Beyotime, P0010) [33]. ALP activity was represented by the $\mathrm{OD}_{405 \mathrm{~nm}}$ value after normalization to the total cellular protein.

\section{Alizarin red $\mathrm{S}$ staining and mineralization assay}

After 21 days of osteogenic induction, calcium deposition was determined by alizarin red S (ARS) staining and semi-quantitative analysis [33]. The cells were fixed with $4 \%$ paraformaldehyde for 15 min and then stained 
with $0.1 \%$ ARS (pH 4.2, Sigma-Aldrich) for $30 \mathrm{~min}$. The stained cells were observed under an inverted microscope (Olympus). The stained mineralized nodules were incubated with $100 \mathrm{mM}$ cetylpyridinium chloride in $10 \mathrm{mM}$ sodium phosphate ( $\mathrm{pH} 7.0)$ for $1 \mathrm{~h}$ to quantify the calcification. The calcium deposition was determined by measuring the absorbance at $\mathrm{OD}_{562 \mathrm{~nm}}$ spectrophotometrically.

\section{Osteoclast induction}

For osteoclast induction, $50 \mathrm{ng} / \mathrm{mL}$ RANKL (R\&D) was added to the BMDM culture medium, and the medium was changed every 2 days. After 3 days of culture, mature osteoclasts could be observed and subsequent experiments were performed.

TRAP staining (Sigma-Aldrich) was performed according to the previously described procedure [34]. The cells were fixed with $4 \%$ paraformaldehyde for $15 \mathrm{~min}$, added with TRAP staining solution, and incubated at $37^{\circ} \mathrm{C}$ for $40 \mathrm{~min}$. TRAP-positive cells containing 3 or more nuclei were considered osteoclasts.

\section{Quantitative reverse transcription polymerase chain reaction (qRT-PCR)}

The total RNA of rBMSCs was extracted using Trizol reagent (Invitrogen, Carlsbad, CA, USA) and then reversely transcribed to obtain stable cDNA using PrimeScript ${ }^{\mathrm{TM}}$ RT reagent Kit with gDNA Eraser (TaKaRa Bio, Otsu, Japan). The qRT-PCR was performed using SYBR Premix Ex Taq II (TaKaRa Bio) in Quant Studio ${ }^{\text {TM }} 3$ real-time fluorescent quantitative PCR instrument (ThermoFisher Scientific, China). Glyceraldehyde 3-phosphate dehydrogenase (Gapdh) was used as an internal reference to normalize the gene expression [30]. The result was calculated using the $2^{-\Delta \Delta \mathrm{Ct}}$ method and expressed as a multiple change relative to GAPDH. The primer sequences are summarized in Additional file 2: Table S1.

\section{Flow cytometry}

After 7 days of osteogenic induction, rBMSCs were harvested in Dulbecco's phosphate buffered saline and incubated for $15 \mathrm{~min}$ at $4{ }^{\circ} \mathrm{C}$ with fluorescent dyes conjugated anti-rat CD29, CD44, CD34 and CD45 (BD Biosciences) protected from light. The apoptosis in N0, H0, $\mathrm{H} 1$, and $\mathrm{H} 5$ group was determined to evaluate the effect of $\operatorname{PPAR} \beta / \delta$ on cell apoptosis in high-glucose environment. After 7 days of culture, the cells in each group were digested and centrifuged. The cells were resuspended in $500 \mu \mathrm{L}$ of the binding buffer and stained with Annexin V to label the apoptotic cells for $10 \mathrm{~min}$. Then, the PI solution was added to label the dead cells according to the PI-Annexin V apoptosis detection kit (BD Bioscience) [35]. After adjusting the cell suspension volume to $1 \mathrm{~mL}$, the samples were assessed on a flow cytometer (ThermoFisher Scientific).

\section{Western blot}

After 7 days of osteogenic induction, the total proteins were extracted by RIPA buffer (Pierce, Rockford, IL) on ice. Equal quantities of protein samples were separated by electrophoresis on 12\% SDS-PAGE polyacrylamide gels. Then, the samples were electro-transferred to PVDF membranes $(0.22 \mu \mathrm{m}$, Millipore $)$ using a wet transfer apparatus (Bio-Rad) and blocked with 5\% BSA in PBS for $1 \mathrm{~h}$ at room temperature. The membranes were incubated overnight at $4{ }^{\circ} \mathrm{C}$ with primary antibodies of AMPK (\#ET1608-40, huabio, 1:1000), p-AMPK (\#2535 T, CST, 1:1000), mTOR (\#ET1608-5, huabio, 1:1000), p-mTOR (\#ABP50363, abbkine, 1:1000), p62 (\#R1309-8, huabio, 1:1000), LC3BI/II (\#ET1701-65, huabio, 1:1000) and $\beta$-ACTIN(\#MA1210-1, huabio, 1:2000), respectively. After that, the blots were incubated with horseradish peroxidase (HRP)-conjugated secondary antibodies (\#HA1001, huabio, 1:5000) at room temperature for $1 \mathrm{~h}$ [30]. The immobilon reagents (Millipore) were used for the visualization and detection of antibody-antigen complexes. The band intensity was measured by ImageJ software.

\section{RNA-seq and gene set enrichment analysis}

The rBMSCs cultured in high-glucose osteogenic differentiation medium for 7 days were collected for RNA sequencing. Trizol reagent (Invitrogen) was used to extract the total RNA. Sequencing library was prepared according to the steps recommended by the Illumina TrueSeq mRNA sample preparation kit. Sequencing was performed on Illumina HiSeq 3000. STAR software was used to map the reads to the rat genome to obtain a gene expression matrix. DESeq2 package was used to perform the differential gene analysis. Genes with adjusted $\mathrm{p}$ value lower than 0.01 and $\mid \log 2$ FoldChange| higher than 1.5 were considered statistically significant. KEGG enrichment analysis was performed using the online analysis tool metascape (http://metascape.org/). Gene Set Enrichment Analysis (GSEA) was carried out through GSEA software (http://www.broad.mit.edu/GSEA, v.4.1.0). The two gene sets of "osteogenesis" and "positive regulation of autophagy" were enriched and analyzed.

\section{Adenoviral infection}

The adenoviral RFP-GFP-LC3 (Hanbio, Shanghai, China) was used to transfect rBMSCs to indicate autophagy flux. In brief, $20 \mathrm{MOI}$ virus solution was added to each well on the 5th day since osteogenic induction. After a 6-h infection, the medium containing virus solution was replaced by fresh culture medium. After another 48 -h incubation, 
rBMSCs were washed with PBS and fixed with $4 \%$ paraformaldehyde for $15 \mathrm{~min}$. DAPI (Invitrogen) was used to mark nuclei. Autophagosomes were visualized by a laser confocal microscope (LSCM, Olympus).

\section{Transmission electron microscope (TEM)}

The number and morphology of autophagosome were observed using a transmission electron microscope (TEM, Hitachi, Japan). Briefly, the cells of each group were digested and centrifuged. Then, the cell mass at the bottom was collected and fixed with $2.5 \%$ glutaraldehyde. After fixation, the cell mass was treated with $1 \%$ osmium acid for $2 \mathrm{~h}$, and dehydrated by ethanol and acetone. Then the sample was embedded with epoxy resin to prepare ultrathin slice $(50-70 \mathrm{~nm})$. The slice was stained with uranyl acetate-lead citrate, and the autophagosome was observed by TEM.

\section{Statistical analysis}

All quantified data were expressed as mean \pm standard deviation (SD). Statistical differences were performed via one-way or two-way analysis of variance (ANOVA) followed by the Tukey's post hoc test for multiple comparisons. $p<0.05$ was considered to be statistically significant.

\section{Results}

\section{PPAR $\beta / \delta$ agonist promotes osteogenic differentiation} and decreases the apoptosis in high-glucose environment The rBMSCs characterization was performed by threeline differentiation and flow cytometry. ARS, Alcian Blue and Oil Red O staining were conducted to evaluate the osteogenic, chondrogenic and adipogenic differentiation potential of rBMSCs (Additional file 1: Figure S1A-C). Flow cytometry showed that the rBMSCs were CD29/ CD44 positive and CD34/CD45 negative (Additional file 1: Figure S1D).

First, we explored the effects of PPAR $\beta / \delta$ agonist on the osteogenic differentiation of rBMSCs in highglucose condition. The ALP staining of rBMSC in HG group was lighter than that in NG group. The staining depth increased with addition of GW501516 in a concentration-dependent manner (Fig. 1A, B). Similar results were observed in ARS staining (Fig. 1C, D). The mRNA expressions of osteogenic markers were decreased in high-glucose medium. The addition of GW501516 partially restored the mRNA expressions of osteogenic markers (Fig. 1E). To investigate whether $\mathrm{PPAR} \beta / \delta$ agonist-mediated promotion of osteogenic differentiation of rBMSCs was specific to GW501516, we explored the osteogenic effect of another PPAR $\beta / \delta$ agonist, GW0742, in rBMSCs. Similar to GW501516, GW0742 increased ALP staining depth and mRNA expressions of osteogenesis-related genes in normal- and high-glucose conditions (Additional file 1: Figure S2A-D). The addition of PPAR $\beta / \delta$ antagonist GSK0660 inhibited the osteogenic differentiation of rBMSCs in normal- and high-glucose conditions (Additional file 1: Figure S2E-G). The high-glucose condition enhanced the apoptotic cells from $6.36 \% \pm 2.11 \%$ to $24.25 \% \pm 3.04 \%$, which was reduced to $17.03 \% \pm 1.79 \%$ and $11.22 \% \pm 1.62 \%$ by adding $1 \mu \mathrm{M}$ and $5 \mu \mathrm{M}$ of GW501516, respectively (Fig. 1F).

In addition, we explored the regulatory effect of $\operatorname{PPAR} \beta / \delta$ agonist on osteoclast differentiation in normaland high-glucose conditions. As previously reported, high-glucose environment inhibited osteoclast differentiation in vitro [36]. Trap staining results showed that there is no significant difference on the number and size of osteoclasts before and after PPAR $\beta / \delta$ agonist treatment in vitro (Additional file 1: Figure S3A, B).

\section{PPAR $\beta / \delta$ agonist improves the bone regeneration of calvarial defects in diabetic rats}

We explored the effects of PPAR $\beta / \delta$ agonist on the bone regeneration of rat calvarial defects (Fig. 2A). Through $\mu \mathrm{CT}$ analysis of calvarial defects, we found that both $\mathrm{BMD}$ and $\mathrm{BV} / \mathrm{TV}$ were reduced in diabetic rats compared with the control, which were significantly increased after injection of GW501516 (Fig. 2B, C). H\&E and Masson staining showed that diabetes mellitus inhibited the formation of new bone and collagen around the calvarial defects, which were promoted by GW501516 (Fig. 3A, B).

\section{The activation of PPAR $\beta / \delta$ in high-glucose environment promotes the autophagy and up-regulates osteogenic pathways}

RNA sequencing analysis was performed on rBMSCs after 7 days of osteogenic induction in high-glucose environment. Compared to H0 group, H5 group had 684 upregulated genes and 420 down-regulated genes (Fig. 4A). KEGG functional enrichment analysis demonstrated that the PPAR-related pathway and AMPK pathway were significantly up-regulated in $\mathrm{H} 5$ group when compared to H0 group, KEGG network diagram showed the close interaction between the PPAR signaling pathway and the AMPK signaling pathway after GW501516 treatment (Fig. 4B, C). The expression of osteogenic marker genes and autophagy pathway-related genes was upregulated in $\mathrm{H} 5$ group (Fig. 4D, E). GSEA enrichment analysis revealed that the osteogenic differentiation and autophagy-related regulatory genes of rBMSCs were 

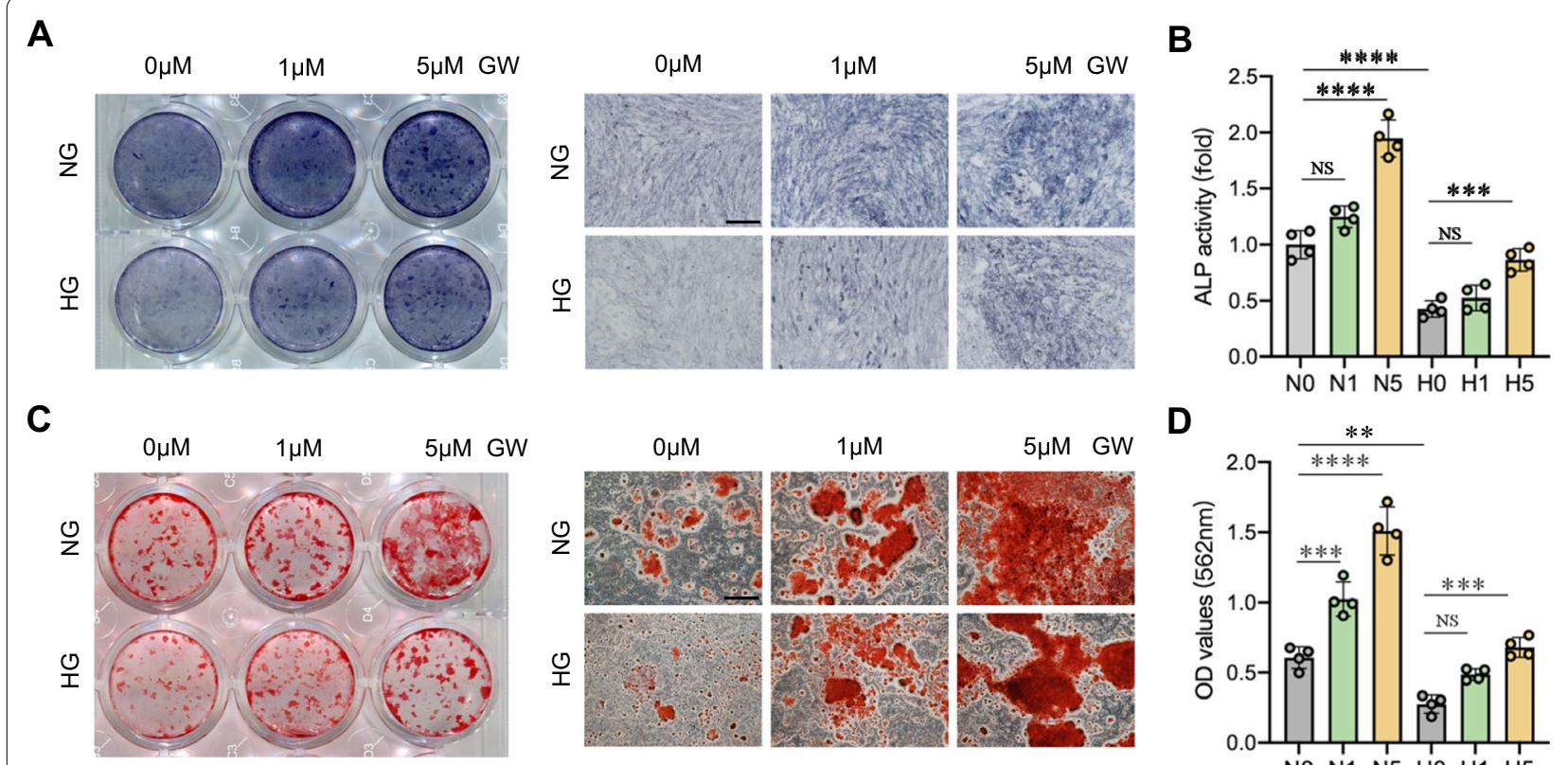

D

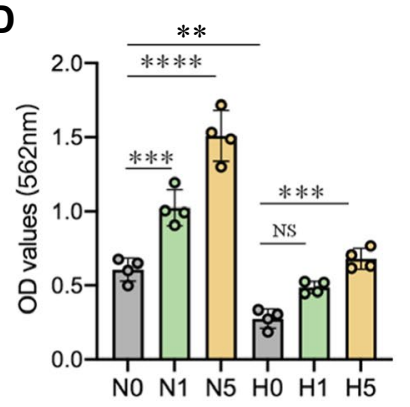

E
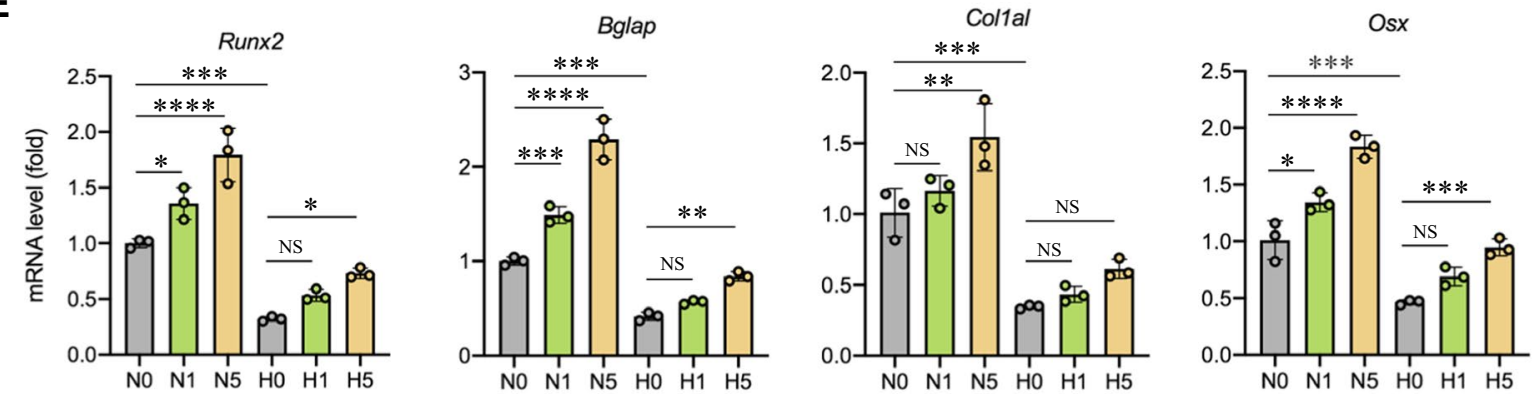

$\mathbf{F}$
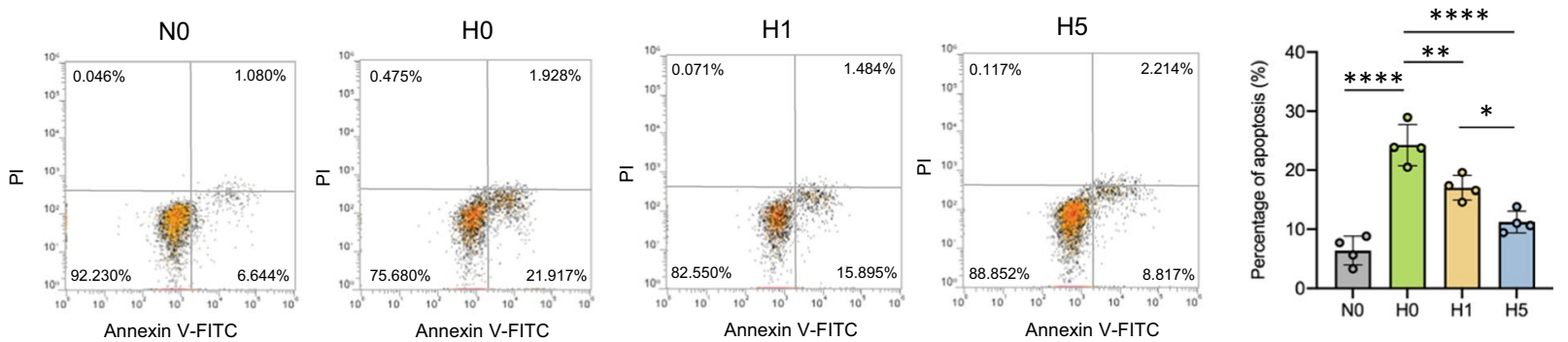

Fig. 1 PPARß/ $\delta$ agonist promotes osteogenic differentiation and decreases the apoptosis in high-glucose environment. A, B ALP staining and ALP activity quantitative analyses of rBMSCs after 7 days of osteogenic induction treated with GW501516 of different concentration (scale bar = 500 $\mu \mathrm{m})$. C, D ARS staining and quantitative analyses of rBMSCs after 21 days of osteogenic induction treated with GW501516 of different concentration (scale bar $=500 \mu \mathrm{m}$ ). E qRT-PCR for the osteogenesis-related genes Runx2, Bglap, Col1a1 and Osx after 7 days of osteogenic induction. F Analysis of cells stained with Annexin V/PI by flow cytometry. Data are expressed as mean \pm SD. The $p$ values were calculated by two-way ANOVA with Tukey's post hoc test. (NS, not statistically significant, ${ }^{*} p<0.05,{ }^{* *} p<0.01,{ }^{* * *} p<0.001,{ }^{* * * *} p<0.0001$ )

(See figure on next page.)

Fig. 2 PPARß $/ \delta$ agonist accelerates bone regeneration of calvarial defects in diabetic rats. A Schematic illustration of experimental design. B Representative images of $\mu C T$ regeneration of carvarial defects at 4 and 8 weeks (scale bar $=3 \mathrm{~mm}$ ). C Quantitative $\mu C T$ analyses of bone mineral density (BMD, $\mathrm{mg} / \mathrm{cc}$ ) and bone volume/total volume (BV/TV, \%) within the original defect position boundary $(\mathrm{n}=8)$. Data are expressed as mean \pm SD. The $p$ values were calculated by two-way ANOVA with Tukey's post hoc test. (NS, not statistically significant, ${ }^{*} p<0.05,{ }^{* * *} p<0.001$, $\left.{ }^{* * * *} p<0.0001\right)$ 
A
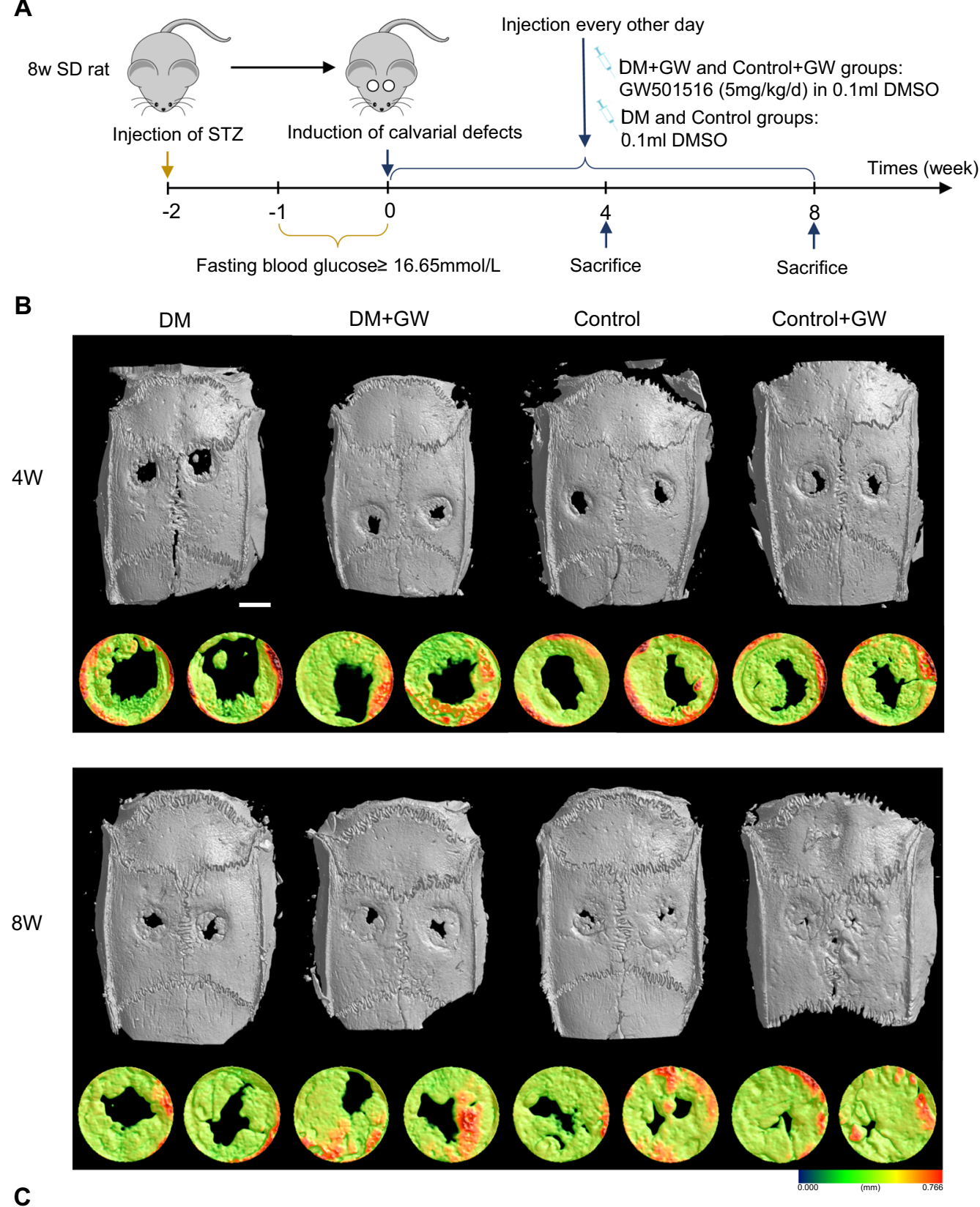

C

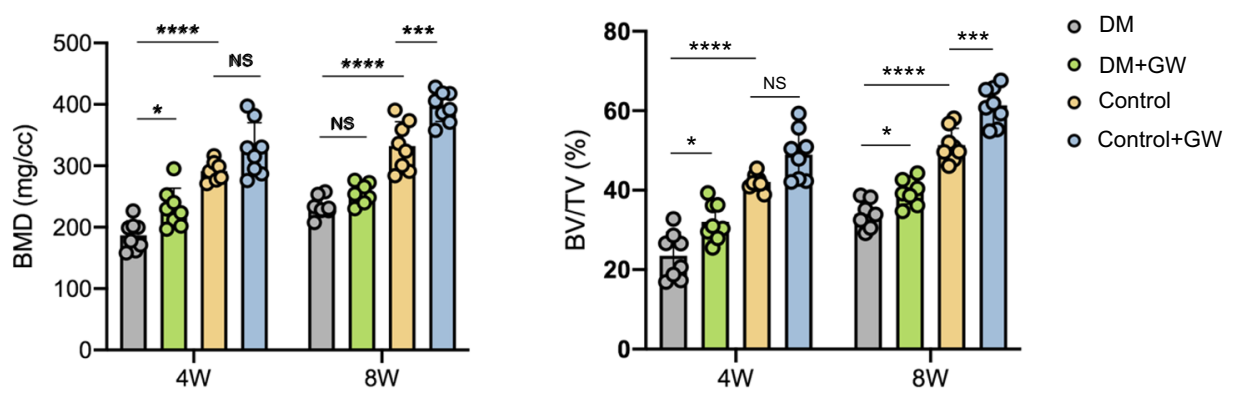

Fig. 2 (See legend on previous page.) 


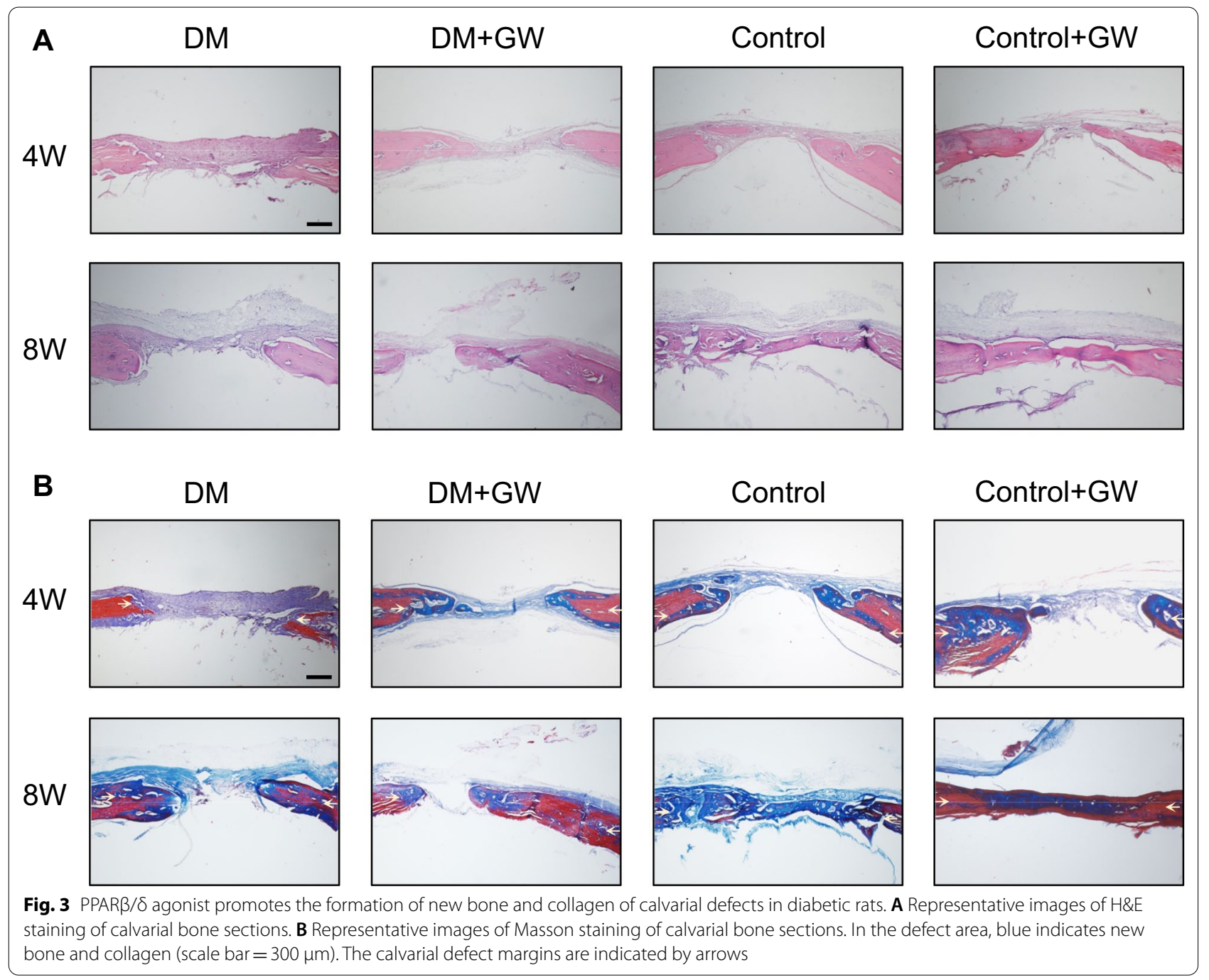

up-regulated by $\mathrm{PPAR} \beta / \delta$ agonist in high-glucose environment (Fig. 4F).

\section{PPAR $\beta / \delta$ agonist promotes AMPK/mTOR-mediated autophagy in high-glucose environment}

The expression of $\mathrm{p}$-AMPK was decreased while $\mathrm{p}-\mathrm{mTOR}$ was increased in $\mathrm{HO}$ group. The addition of GW501516 enhanced p-AMPK level and reduced p-mTOR level. Compared to N0 group, the reduction in p62 expression and increase in LC3-II/LC3-I ratio were detected in $\mathrm{H} 0$ group, both of which were amplified in $\mathrm{H} 5$ group (Fig. $5 \mathrm{~A}, \mathrm{~B}$ ), the increase in the ratio of LC3-II/LC3-I and the decrease in p62 protein level can reflect the activation in autophagy [56]. The observation from LSCM and TEM showed that the number of autophagosomes was enhanced in $\mathrm{H} 0$ group, which was further elevated in $\mathrm{H} 5$ group (Fig. 5C, D).
PPAR $\beta / \delta$ agonist promotes osteogenic differentiation of rBMSCs through AMPK/mTOR-regulated autophagy We used 3-Methyladenine (3-MA), a specific autophagy inhibitor, to identify the involvement of autophagy in GW501516-mediated osteogenic differentiation. The degree of ALP staining and mineralized nodules of ARS staining was decreased by adding 3-MA. The increased osteogenic differentiation by GW501516 was also partially inhibited (Fig. 6A, B). Compared to H5 group, the mRNA expressions of osteogenic markers were decreased by adding 3-MA (Fig. 6C). Then, we used AMPK inhibitor Compound $\mathrm{C}$ to further investigate the connection between PPAR $\beta / \delta$ and AMPK signal pathway. The ALP staining degree of rBMSCs in GW501516+Compound $\mathrm{C}(\mathrm{H} 5+$ Compound $\mathrm{C}$ ) group was significantly decreased. And the addition of Compound $\mathrm{C}$ inhibited mRNA expressions of osteogenesis-related genes (Additional file 1: Figure S4A-C). 


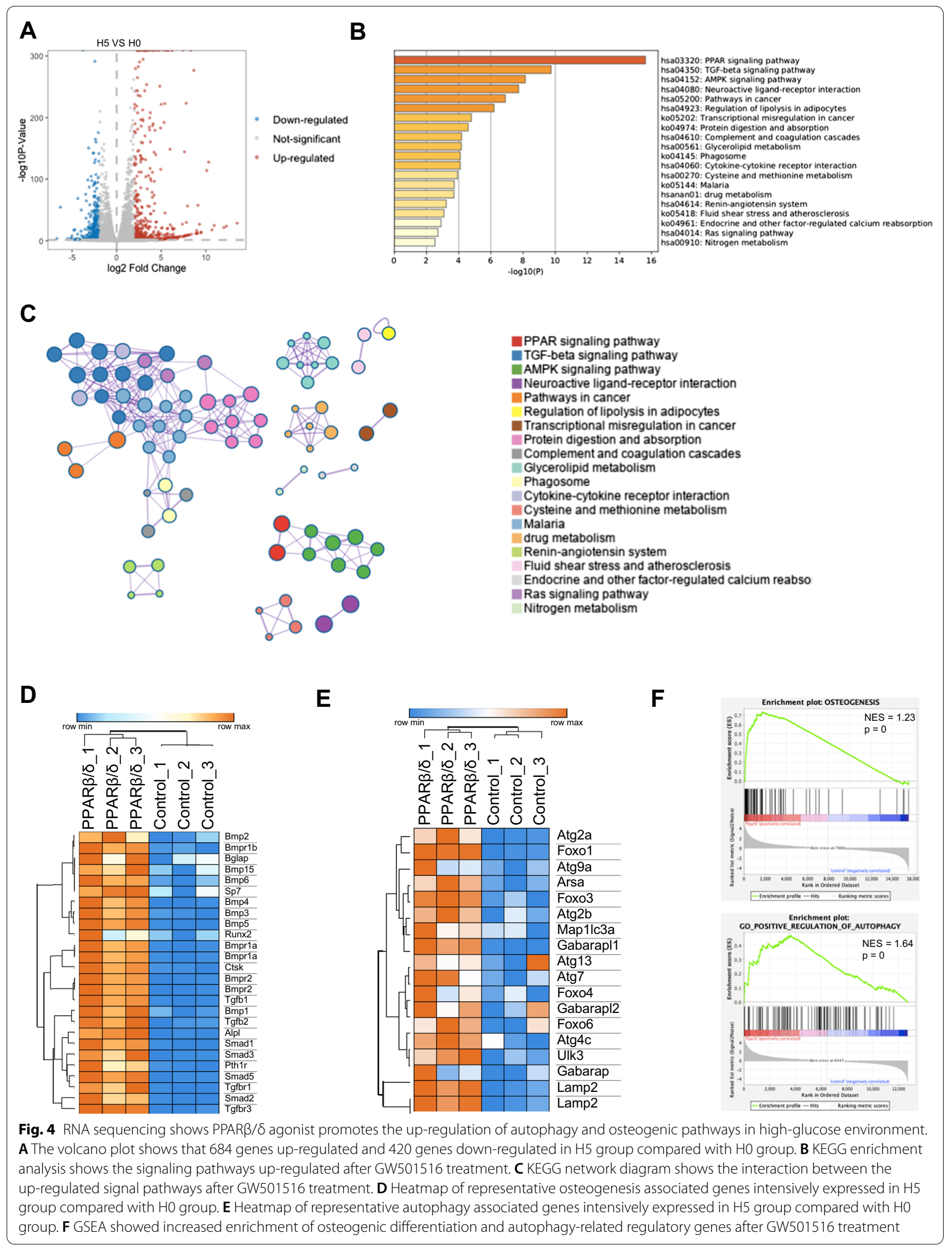




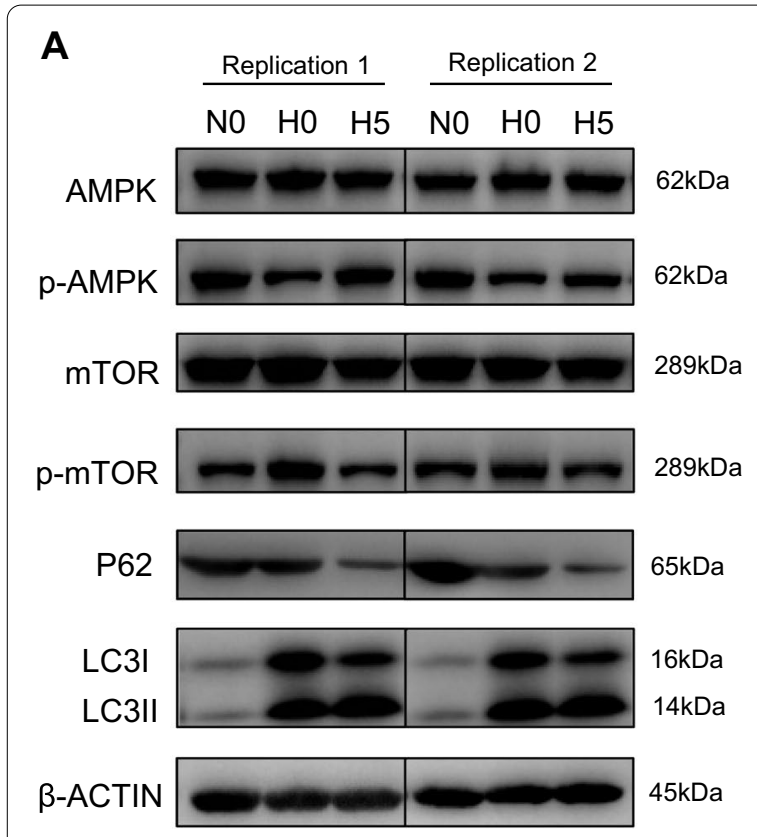

B
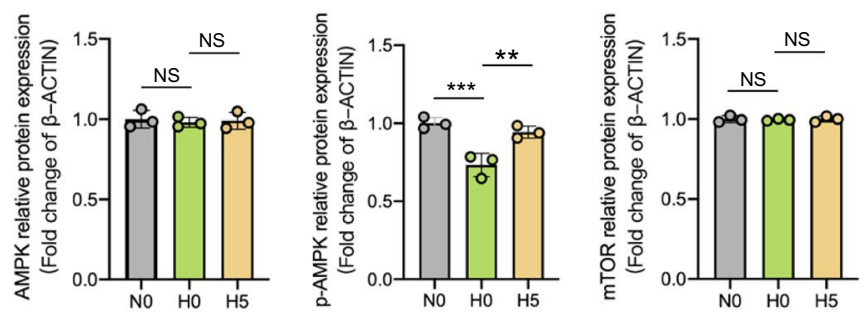

C
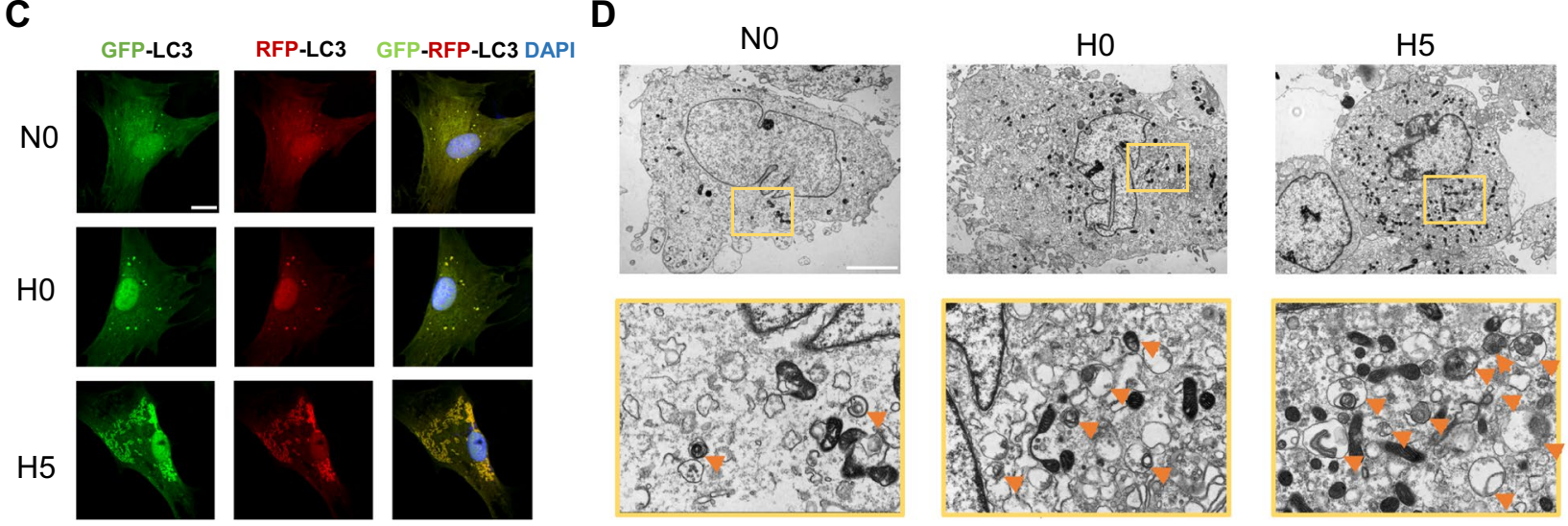

Fig. 5 PPARß/ $\delta$ agonist promotes AMPK/mTOR-mediated autophagy in high-glucose environment. A AMPK, p-AMPK, mTOR, p-mTOR, LC3 and p62 were resolved via western blot after $\mathrm{NO}, \mathrm{H} 0$ and $\mathrm{H} 5$ groups cultured for 7 days of osteogenic induction. $\beta$-ACTIN was the loading control. B Gray values of bands of AMPK, p-AMPK, mTOR, p-mTOR, LC3 and p62. C LSCM images of rBMSCs transfected with RFP-GFP-LC3 adenovirus. DAPI labeled the nuclei (scale bar $=16 \mu \mathrm{m}$ ). The merge number of RFP and GFP dot formation (yellow) indicated the autophagy level. D TEM images of rBMSCs after 7 days of osteogenic induction in high-glucose environment (scale bar $=50 \mu \mathrm{m}$ ). Typical autophagosomes are indicated by arrows. Data are expressed as mean \pm SD. The $p$ values were calculated by one-way ANOVA with Tukey's post hoc test. (NS, not statistically significant, ${ }^{*} p<0.05$, $\left.{ }^{* *} p<0.01,{ }^{* * *} p<0.001,{ }^{* * * *} p<0.0001\right)$

\section{Discussion}

The long-term exposure to diabetes mellitus disturbs bone metabolism and leads to impaired bone quality, which predispose diabetic patients to higher fracture risk and slower osseus healing [6]. Currently, it is still clinically challenging to achieve ideal bone healing in diabetic patients [9]. Recent studies revealed that PPAR $\beta / \delta$ served as a critical regulator of bone metabolism and promoted the osteogenic differentiation of MSCs. Additionally, the use of PPAR $\beta / \delta$ agonists has been shown to alleviate the progression of diabetic mellitus [37-39]. Therefore, the present study investigated the effects of PPAR $\beta / \delta$ agonists on the osteogenic differentiation of rBMSCs in vitro and on the bone healing of calvarial defects in vivo under high-glucose condition.

Consistent with previous studies [40], the osteogenic differentiation of rBMSCs was inhibited in high-glucose environment in our study, while the inhibitory effect was partially reversed by PPAR $\beta / \delta$ agonist GW501516 in a concentration-dependent 


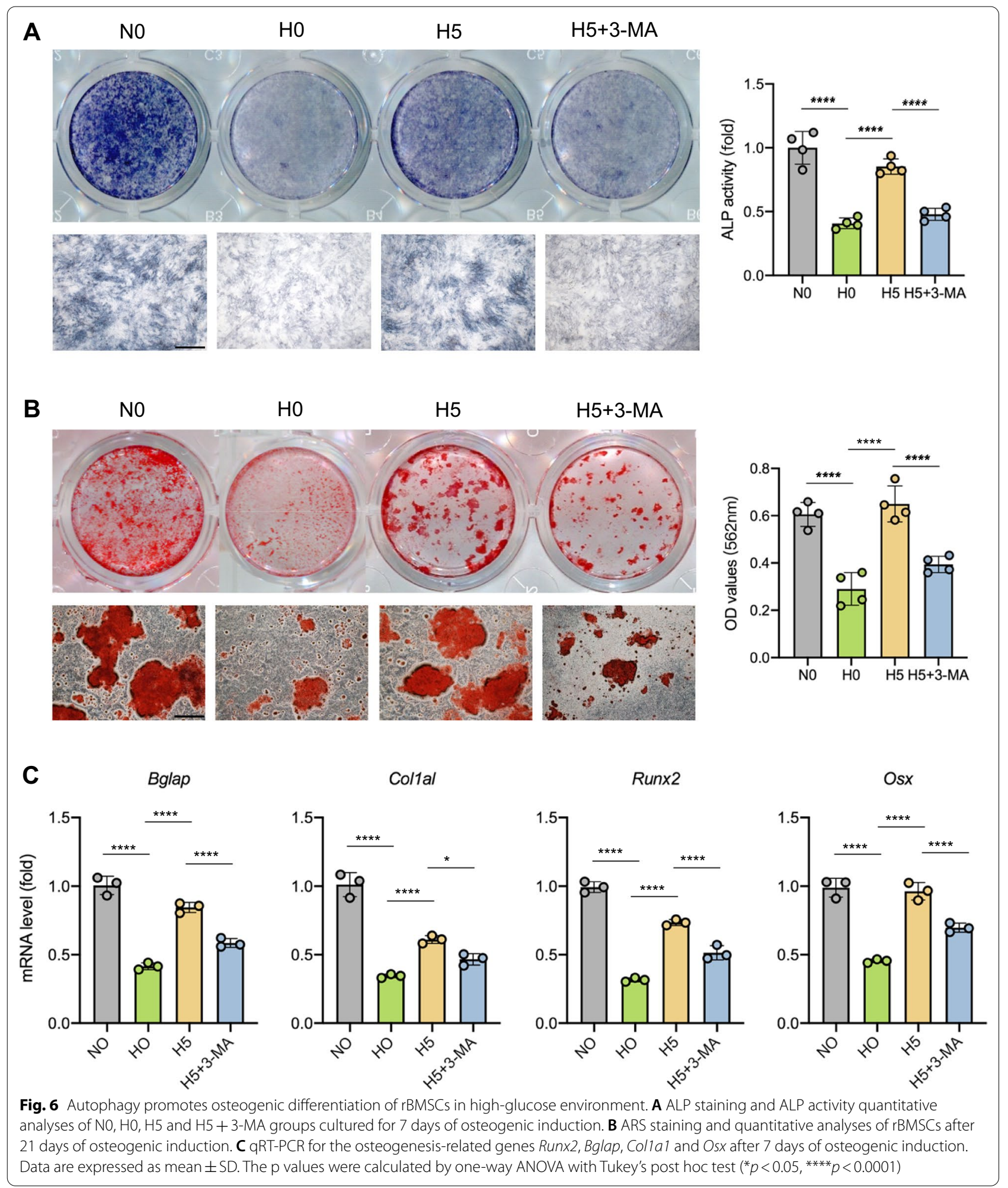

manner. In addition, the delayed bone regeneration of calvarial defects in diabetic rats was also promoted when treated with GW501516. The beneficial effects of PPAR $\beta / \delta$ on bone formation were recently revealed. In normal-glucose environment, $\operatorname{PPAR} \beta / \delta$ enhanced the osteogenic differentiation of MSCs via 
Wnt signal pathway and restored bone density in a mouse model of postmenopausal osteoporosis [19]. The PPAR $\beta / \delta$-induced osteogenesis was also related to peroxisome expression and redox balance [22]. However, the mechanisms of PPAR $\beta / \delta$-mediated osteogenesis in high-glucose environment remained unclear. Hence, we performed RNA sequencing to explore the mechanisms of PPAR $\beta / \delta$-mediated osteogenesis in hyperglycemia condition. The KEGG analysis found that the AMPK signaling pathway was up-regulated by GW501516, and the subsequent GESA analysis suggested that both osteogenesis and autophagy activity were up-regulated. The involvement of AMPK pathway and autophagy in osteogenesis has been well documented [41-43]. Briefly, AMPK is a critical signal molecule that maintains the homeostasis of cell metabolism [44]. The activation of AMPK inhibits mTOR phosphorylation, thereby triggering autophagy [45-49]. Autophagy regulates the stemness of MSCs and is a necessary process in osteogenic differentiation and mineralization [50]. Hence, the PPAR $\beta / \delta$-induced osteogenesis in high-glucose environment is likely to be caused by the AMPK pathway-mediated autophagy.

Subsequently, we investigated the expression of autophagy-related protein and formation of autophagosomes. It was not surprising to detect enhanced autophagy in HG group. As an adaptive response, autophagy can be activated by various pathological environments to maintain cellular homeostasis and clear damaged organelles and misfolded proteins via autolysosome degradation pathway [51]. However, the reduction in $\mathrm{p}$-AMPK level and the resulting increase in p-mTOR level in HG group did not lead to the inhibition of autophagy, indicating that the high-glucose condition suppressed the activation of AMPK, and the AMPK/mTOR axis was not the critical regulator of autophagy in high-glucose environment without other interventions. Indeed, numerous studies found that high-glucose condition enhanced autophagy while reducing p-AMPK and increasing p-mTOR [42, 5255]. In this study, the addition of GW501516 greatly enhanced p-AMPK level, yielding decreased mTOR level, which further increased autophagy. This finding was consistent with previous studies demonstrating the regulatory effect of AMPK/mTOR pathway on autophagy [56, 57]. These results also demonstrated that the activation of PPAR $\beta / \delta$ promoted autophagy in MSCs in high-glucose environment via AMPK/mTOR pathway.

3-Methyladenine (3-MA) was used to further verify whether the autophagy was involved in the PPAR $\beta / \delta$ induced osteogenesis in high-glucose environment.
3-MA is an effective inhibitor of autophagy by blocking autophagy vesicle initiation-related PI3K/AKT pathway [58]. The osteogenic effect of cells under GW501516 treatment in high-glucose condition was suppressed by $3-\mathrm{MA}$, which proved the involvement of autophagy in PPAR $\beta / \delta$-induced osteogenesis. By administering the AMPK inhibitor Compound $C$, we investigated the role of AMPK signaling pathway in PPAR $\beta / \delta$-mediated osteogenic differentiation. Inhibition of AMPK almost completely eliminated the restored osteogenic differentiation induced by $\mathrm{PPAR} \beta / \delta$ agonist under high-glucose condition. Taken together, it seems reasonable to speculate that $\mathrm{PPAR} \beta / \delta$ promoted the osteogenesis in high-glucose environment via AMPK/mTOR-mediated autophagy.

\section{Conclusion}

The present study demonstrates that the activation of $\operatorname{PPAR} \beta / \delta$ can improve the osteogenic differentiation of rBMSCs in high-glucose environment and promote the healing of calvarial defects in diabetic rats for the first time. To summarize, the PPAR $\beta / \delta$ activation up-regulates AMPK phosphorylation, which leads to mTOR suppression and enhanced autophagy activity. Thus, the impaired osteogenic differentiation of MSCs in highglucose condition can be restored. Our data suggest $\mathrm{PPAR} \beta / \delta$ to be a potential molecular target for the treatment of impaired bone quality and delayed bone regeneration in diabetic patients.

\section{Abbreviations}

PPAR $\beta / \delta$ : Peroxisome proliferators-activated receptor $\beta / \delta$; rBMSCs: Rat bone marrow mesenchymal stem cells; ALP: Alkaline phosphatase; ARS: Alizarin red S; qRT-PCR: Quantitative reverse transcription polymerase chain reaction; DM: Diabetes mellitus group; DM + GW: Diabetes mellitus treated with PPARß/ $\delta$ agonist GW501516 group; Control: Control group; Control + GW: Control treated with GW501516; NG: Normal glucose; HG: High glucose; N0: 5.6 mM glucose; N1: 5.6 mM glucose + 1 HM GW501516; N5: 5.6 mM glucose + $5 \mu \mathrm{M}$ GW501516; H0: $30 \mathrm{mM}$ glucose; $\mathrm{H1}: 30 \mathrm{mM}$ glucose $+1 \mu \mathrm{M}$ GW501516; H5: 30 mM glucose + 5 HM GW501516; LSCM: Laser confocal microscope; TEM: Transmission electron microscope; $\mu C T$ : Micro-computed tomography; AMPK: Adenosine 5'-monophosphate-activated protein kinase; mTOR: Mammalian target of rapamycin; MSC: Mesenchymal stem cell; ROS: Reactive oxygen species; PPREs: Peroxisome proliferator response elements; SD: Sprague-Dawley; STZ: Streptozotocin; DMSO: Dimethyl sulfoxide; ROI: Region of interest; BMD: Bone mineral density; BV/TV: Bone volume/total volume; GAPDH: Glyceraldehyde 3-phosphate dehydrogenase; RUNX2: Runtrelated transcription factor 2; Bglap: Bone gamma-carboxyglutamate (gla) protein; Col1a1: Collagen, type I, alpha 1; Osx: Osterix; H\&E: Hematoxylin and eosin; EDTA: Ethylene diamine tetraacetic acid; DMEM: Dulbecco's modification of Eagle's medium; RIPA: Radio-immunoprecipitation assay; SDS-PAGE: Sulfate-polyacrylamide gel electrophoresis; RNA-seq: RNA sequencing; KEGG: Kyoto encyclopedia of genes and genomes; GSEA: Gene set enrichment analysis; ANOVA: Analysis of variance; 3-MA: 3-Methyladenine; SD: Standard deviation. 


\section{Supplementary Information}

The online version contains supplementary material available at https://doi. org/10.1186/s13287-021-02628-8.

Additional file 1: Figure S1. The rBMSCs characterization was performed by three-line differentiation and flow cytometry. (A) ARS staining after osteogenic induction (scale bar $=500 \mu \mathrm{m}$ ). (B) Alcian Blue staining after chondrogenic induction (scale bar $=50 \mu \mathrm{m}$ ) (C) Oil Red O after adipogenic induction (scale bar $=50 \mu \mathrm{m}$ ) (D) rBMSCs were CD29/CD44 positive and CD34/CD45 negative. Figure $\mathbf{S 2}$. The effects of PPAR $\beta / \delta$ agonist (GW0742) and antagonist (GSK0660) on the osteogenic differentiation of rBMSCs in normal- and high-glucose conditions. (A, B) ALP staining and ALP activity quantitative analyses of rBMSCs after 7 days of osteogenic

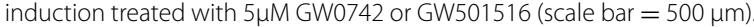
(C) qRT-PCR for the osteogenesis-related genes Runx2, Bglap, Col1a1 and Osx after 7 days of osteogenic induction. (D) qRT-PCR for Bmp2 after 7 days of osteogenic induction. (E, F) ALP staining and ALP activity quantitative analyses of rBMSCs after 7 days of osteogenic induction treated with $1 \mu \mathrm{M}$ GSK0660 (scale bar $=500 \mu \mathrm{m}$ ). (G) qRT-PCR for the osteogenesis-related genes Runx2, Bglap, Colla1 and Osx after 7 days of osteogenic induction. Data are expressed as mean \pm SD. The $p$ values were calculated by twoway ANOVA with Tukey's post hoc test. (NS, not statistically significant, $\left.{ }^{*} p<0.05,{ }^{* *} p<0.01,{ }^{* * *} p<0.001,{ }^{* * * *} p<0.0001\right)$. Figure $\mathbf{S 3}$. The effect of GW501516 on osteoclast differentiation of bone marrow-derived macrophage (BMDM) in normal- and high-glucose conditions. (A) TRAP staining for osteoclast differentiation before and after PPAR $/ \delta$ agonist treatment (Scale bar $=100 \mu \mathrm{m}$ ). (B) Statistics on the number and size of osteoclasts. Data were expressed as mean $\pm \mathrm{SD}$. The $p$ values were calculated by two-way ANOVA with Tukey's post hoc test. (NS, not statistically significant). N.OC = number of osteoclasts. Figure S4. The effects of AMPK inhibitor (Compound C) plus PPARB/ $\delta$ agonist (GW501516) on the osteogenic differentiation of rBMSCs in high-glucose conditions. (A, B) ALP staining and ALP activity quantitative analyses of rBMSCs after 7 days of osteogenic induction treated with $5 \mu \mathrm{M}$ GW501516 + 1 $\mu \mathrm{M}$ Compound $C$ (scale bar $=500 \mu \mathrm{m})$. (C) qRT-PCR for the osteogenesis-related genes Runx2, Bglap, Col1a1, and Osx after 7 days of osteogenic induction. Data are expressed as mean \pm SD. The $p$ values were calculated by two-way ANOVA with Tukey's post hoc test. $\left.{ }^{* *} p<0.01,{ }^{* * *} p<0.001,{ }^{* * * *} p<0.0001\right)$.

Additional file 2. The primer sequences for $\mathrm{QRT}$-PCR.

\section{Acknowledgements}

Not applicable.

\section{Authors' contributions}

JRY and ZHZ designed the study. MC, DJ and RY performed the experiments and were involved in data analyses and interpretation. MC, JRY and ZHZ wrote and revised the manuscript. All authors read and approved the final manuscript.

\section{Funding}

This work was supported by grants from National Natural Science Foundation of China (grant no. 81801018, 81400522, 81771048 and 81901041), and Sichuan Science and Technology Program (Grant No. 2020YFS0170).

\section{Availability of date and materials}

The datasets used during the current study are available from the corresponding authors on reasonable request.

\section{Declarations}

\section{Ethics approval and consent to participate}

The animal studies were approved by the Animal Care and Ethics Committee of West China Hospital of Stomatology, Sichuan University (WCHSIRB-D-2018-057).

\section{Consent for publication}

Not applicable.

\section{Competing interests}

The authors declare that they have no competing interests.

\section{Author details}

${ }^{1}$ State Key Laboratory of Oral Diseases and National Clinical Research Center for Oral Diseases, West China Hospital of Stomatology, Sichuan University, Chengdu, China. ${ }^{2}$ Department of Orthodontics, West China Hospital of Stomatology, Sichuan University, No. 14, 3rd Section, South Renmin Road, Chengdu 610041, Sichuan, China. ${ }^{3}$ Department of Orthodontics, Shanghai Ninth People's Hospital, Collage of Stomatology, Shanghai Jiao Tong University School of Medicine, Shanghai, China.

Received: 8 June 2021 Accepted: 16 October 2021

Published online: 04 November 2021

\section{References}

1. Howard SG, Sargis RM. Type 1 diabetes-origins and epidemiology. Lancet Diabetes Endocrinol. 2020;8(5):367-8.

2. Balakumar P, Maung UK, Jagadeesh G. Prevalence and prevention of cardiovascular disease and diabetes mellitus. Pharmacol Res. 2016;113(Pt A):600-9.

3. Tao T, Xu H. Autophagy and obesity and diabetes. Adv Exp Med Biol. 2020;1207:445-61.

4. Wang L, Gao P, Zhang M, Huang Z, Zhang D, Deng Q, Li Y, Zhao Z, Qin X, Jin $D$, et al. Prevalence and ethnic pattern of diabetes and prediabetes in China in 2013. JAMA. 2017:317(24):2515-23.

5. Bouillon R. Diabetic bone disease. Calcif Tissue Int. 1991;49(3):155-60.

6. Bizzarri C, Benevento D, Giannone G, Bongiovanni M, Anziano M, Patera IP, Cappa M, Cianfarani S. Sexual dimorphism in growth and insulinlike growth factor-l in children with type 1 diabetes mellitus. Growth Hormone IGF Res Off J Growth Hormone Res Soc Int IGF Res Soc. 2014;24(6):256-9

7. Kurra S, Fink DA, Siris ES. Osteoporosis-associated fracture and diabetes. Endocrinol Metab Clin N Am. 2014;43(1):233-43.

8. Lecka-Czernik B. Diabetes, bone and glucose-lowering agents: basic biology. Diabetologia. 2017;60(7):1163-9.

9. Ferrari SL, Abrahamsen B, Napoli N, Akesson K, Chandran M, Eastell R, El-Hajj Fuleihan G, Josse R, Kendler DL, Kraenzlin M, et al. Diagnosis and management of bone fragility in diabetes: an emerging challenge. Osteoporos Int J Establ Result Coop Between Eur Found Osteoporos Natl Osteoporos Found USA. 2018;29(12):2585-96.

10. Loder RT. The influence of diabetes mellitus on the healing of closed fractures. Clin Orthop Relat Res. 1988;232:210-6.

11. Choi WJ, Lee JS, Lee M, Park JH, Lee JW. The impact of diabetes on the short- to mid-term outcome of total ankle replacement. Bone Joint J. 2014;96-b(12):1674-80.

12. Wang J, Wang B, Li Y, Wang D, Lingling E, Bai Y, Liu H. High glucose inhibits osteogenic differentiation through the BMP signaling pathway in bone mesenchymal stem cells in mice. Excli J. 2013;12:584-97.

13. Zhang D, Lu H, Chen Z, Wang Y, Lin J, Xu S, Zhang C, Wang B, Yuan Z, Feng $X$, et al. High glucose induces the aging of mesenchymal stem cells via Akt/mTOR signaling. Mol Med Rep. 2017;16(2):1685-90.

14. Zhao W, Zhang W-L, Yang B, Sun J, Yang M-W. NIPA2 regulates osteoblast function via its effect on apoptosis pathways in type 2 diabetes osteoporosis. Biochem Biophys Res Commun. 2019;513(4):883-90.

15. Sanches $C P$, Vianna AGD, Barreto FC. The impact of type 2 diabetes on bone metabolism. Diabetol Metab Syndr. 2017;9:85.

16. Magadum A, Engel FB. PPARbeta/delta: linking metabolism to regeneration. Int J Mol Sci. 2018;19(7):2013.

17. Liu Y, Colby JK, Zuo X, Jaoude J, Wei D, Shureiqi I. The role of PPAR- $\delta$ in metabolism, inflammation, and cancer: many characters of a critical transcription factor. Int J Mol Sci. 2018;19(11):3339.

18. Djouad F, Ipseiz N, Luz-Crawford P, Scholtysek C, Kronke G, Jorgensen C. PPARbeta/delta: a master regulator of mesenchymal stem cell functions. Biochimie. 2017;136:55-8.

19. Scholtysek C, Katzenbeisser J, Fu H, Uderhardt S, Ipseiz N, Stoll C, Zaiss MM, Stock M, Donhauser L, Bohm C, et al. PPARbeta/delta governs Wnt signaling and bone turnover. Nat Med. 2013;19(5):608-13. 
20. Müller DIH, Stoll C, Palumbo-Zerr K, Böhm C, Krishnacoumar B, Ipseiz N, Taubmann J, Zimmermann M, Böttcher M, Mougiakakos D, et al. PPARSmediated mitochondrial rewiring of osteoblasts determines bone mass. Sci Rep. 2020;10(1):8428.

21. Fu H, Desvergne B, Ferrari S, Bonnet N. Impaired musculoskeletal response to age and exercise in PPAR $\beta(-/-)$ diabetic mice. Endocrinology. 2014;155(12):4686-96.

22. Qian G, Fan W, Ahlemeyer B, Karnati S, Baumgart-Vogt E. Peroxisomes in different skeletal cell types during Intramembranous and endochondral ossification and their regulation during osteoblast differentiation by distinct peroxisome proliferator-activated receptors. PLoS ONE. 2015;10(12):0143439.

23. Lee CH, Olson P, Hevener A, Mehl I, Chong LW, Olefsky JM, Gonzalez FJ, Ham J, Kang H, Peters JM, et al. PPARdelta regulates glucose metabolism and insulin sensitivity. Proc Natl Acad Sci USA. 2006;103(9):3444-9.

24. Gross B, Pawlak M, Lefebvre P, Staels B. PPARs in obesity-induced T2DM, dyslipidaemia and NAFLD. Nat Rev Endocrinol. 2017;13(1):36-49.

25. Mosti MP, Stunes AK, Ericsson M, Pullisaar H, Reseland JE, Shabestari M, Eriksen EF, Syversen U. Effects of the peroxisome proliferator-activated receptor (PPAR)-delta agonist GW501516 on bone and muscle in ovariectomized rats. Endocrinology. 2014;155(6):2178-89.

26. Wang Y, Chen J, Li S, Zhang X, Guo Z, Hu J, Shao X, Song N, Zhao Y, Li H, et al. Exogenous spermine attenuates rat diabetic cardiomyopathy via suppressing ROS-p53 mediated downregulation of calcium-sensitive receptor. Redox Biol. 2020;32:101514.

27. Hu Z, Ma C, Rong X, Zou S, Liu X. Immunomodulatory ECM-like microspheres for accelerated bone regeneration in diabetes mellitus. ACS Appl Mater Interfaces. 2018;10(3):2377-90.

28. Pei M, Li J, McConda DB, Wen S, Clovis NB, Danley SS. A comparison of tissue engineering based repair of calvarial defects using adipose stem cells from normal and osteoporotic rats. Bone. 2015;78:1-10.

29. Bouxsein ML, Boyd SK, Christiansen BA, Guldberg RE, Jepsen KJ, Müller R. Guidelines for assessment of bone microstructure in rodents using micro-computed tomography. J Bone Miner Res Off J Am Soc Bone Miner Res. 2010;25(7):1468-86.

30. Wang Y, Deng P, Liu Y, Wu Y, Chen Y, Guo Y, Zhang S, Zheng X, Zhou L, Liu $W$, et al. Alpha-ketoglutarate ameliorates age-related osteoporosis via regulating histone methylations. Nat Commun. 2020;11(1):5596.

31. Chen L, Zhang RY, Xie J, Yang JY, Fang KH, Hong CX, Yang RB, Bsoul N, Yang L. STAT3 activation by catalpol promotes osteogenesis-angiogenesis coupling, thus accelerating osteoporotic bone repair. Stem Cell Res Ther. 2021;12(1):108.

32. Tang Y, Zheng L, Zhou J, Chen Y, Yang L, Deng F, Hu Y. miR2033p participates in the suppression of diabetesassociated osteogenesis in the jaw bone through targeting Smad1. Int J Mol Med. 2018;41(3):1595-607.

33. Lin W, Li Q, Zhang D, Zhang X, Qi X, Wang Q, Chen Y, Liu C, Li H, Zhang S, et al. Mapping the immune microenvironment for mandibular alveolar bone homeostasis at single-cell resolution. Bone Res. 2021;9(1):17

34. Liu W, Zhou L, Zhou C, Zhang S, Jing J, Xie L, Sun N, Duan X, Jing W, Liang $X$, et al. GDF11 decreases bone mass by stimulating osteoclastogenesis and inhibiting osteoblast differentiation. Nat Commun. 2016;7:12794.

35. Chen X, Yang X, Yuan P, Jin R, Bao L, Qiu X, Liu S, Liu T, Gooding JJ, Chen $W$, et al. Modular immune-homeostatic microparticles promote immune tolerance in mouse autoimmune models. Sci Transl Med. 2021;13:584.

36. Wittrant Y, Gorin Y, Woodruff K, Horn D, Abboud HE, Mohan S, AbboudWerner SL. High d(+)glucose concentration inhibits RANKL-induced osteoclastogenesis. Bone. 2008;42(6):1122-30.

37. Luquet S, Gaudel C, Holst D, Lopez-Soriano J, Jehl-Pietri C, Fredenrich A, Grimaldi PA. Roles of PPAR delta in lipid absorption and metabolism: a new target for the treatment of type 2 diabetes. Biochem Biophys Acta. 2005;1740(2):313-7.

38. Han L, Shen WJ, Bittner S, Kraemer FB, Azhar S. PPARs: regulators of metabolism and as therapeutic targets in cardiovascular disease-part II: PPAR- $\beta / \delta$ and PPAR- $\gamma$. Future Cardiol. 2017;13(3):279-96.

39. Abdel-Rahman EA, Bhattacharya S, Buabeid M, Majrashi M, Bloemer J, Tao YX, Dhanasekaran M, Escobar M, Amin R, Suppiramaniam V. PPAR- $\delta$ activation ameliorates diabetes-induced cognitive dysfunction by modulating integrin-linked kinase and AMPA receptor function. J Am Coll Nutr. 2019;38(8):693-702

40. Tan J, Zhou L, Zhou Y, Xue P, Wu G, Dong G, Guo H, Wang Q. The influence of diabetes mellitus on proliferation and osteoblastic differentiation of MSCs. Curr Stem Cell Res Ther. 2017;12(5):388-400.

41. Madhavi YV, Gaikwad N, Yerra VG, Kalvala AK, Nanduri S, Kumar A Targeting AMPK in diabetes and diabetic complications: energy homeostasis, autophagy and mitochondrial health. Curr Med Chem. 2019;26(27):5207-29.

42. Kim J, Kundu M, Viollet B, Guan KL. AMPK and mTOR regulate autophagy through direct phosphorylation of Ulk1. Nat Cell Biol. 2011;13(2):132-41.

43. Vidoni C, Ferraresi A, Secomandi E, Vallino L, Gardin C, Zavan B, Mortellaro C, Isidoro C. Autophagy drives osteogenic differentiation of human gingival mesenchymal stem cells. Cell Commun Signal. 2019;17(1):98.

44. Ke R, Xu Q, Li C, Luo L, Huang D. Mechanisms of AMPK in the maintenance of ATP balance during energy metabolism. Cell Biol Int. 2018;42(4):384-92.

45. Han D, Jiang L, Gu X, Huang S, Pang J, Wu Y, Yin J, Wang J. SIRT3 deficiency is resistant to autophagy-dependent ferroptosis by inhibiting the AMPK/mTOR pathway and promoting GPX4 levels. J Cell Physiol. 2020;235(11):8839-51.

46. Alers S, Löffler AS, Wesselborg S, Stork B. Role of AMPK-mTOR-UIk1/2 in the regulation of autophagy: cross talk, shortcuts, and feedbacks. Mol Cell Biol. 2012;32(1):2-11.

47. Wang S, Zhu X, Xiong L, Zhang Y, Ren J. Toll-like receptor 4 knockout alleviates paraquat-induced cardiomyocyte contractile dysfunction through an autophagy-dependent mechanism. Toxicol Lett. 2016;257:11-22.

48. Wang JF, Mei ZG, Fu Y, Yang SB, Zhang SZ, Huang WF, Xiong L, Zhou HJ, Tao W, Feng ZT. Puerarin protects rat brain against ischemia/reperfusion injury by suppressing autophagy via the AMPK-mTOR-ULK1 signaling pathway. Neural Regen Res. 2018;13(6):989-98.

49. Pyo KE, Kim CR, Lee M, Kim JS, Kim KI, Baek SH. ULK1 O-GlcNAcylation Is Crucial for Activating VPS34 via ATG14L during Autophagy Initiation. Cell Rep. 2018;25(10):2878-2890.e2874.

50. Xiao L, Xiao Y. The autophagy in osteoimmonology: self-eating, maintenance, and beyond. Front Endocrinol (Lausanne). 2019;10:490.

51. Choi AM, Ryter SW, Levine B. Autophagy in human health and disease. N Engl J Med. 2013;368(7):651-62.

52. Zhang X, Zhang L, Chen Z, Li S, Che B, Wang N, Chen J, Xu C, Wei C. Exogenous spermine attenuates diabetic kidney injury in rats by inhibiting AMPK/mTOR signaling pathway. Int J Mol Med. 2021;47:3.

53. Chen H, Ji Y, Yan X, Su G, Chen L, Xiao J. Berberine attenuates apoptosis in rat retinal Müller cells stimulated with high glucose via enhancing autophagy and the AMPK/mTOR signaling. Biomed Pharmacother. 2018:108:1201-7.

54. Chang TC, Hsu MF, Wu KK. High glucose induces bone marrow-derived mesenchymal stem cell senescence by upregulating autophagy. PLOS ONE. 2015;10(5):e0126537.

55. Zhao K, Hao H, Liu J, Tong C, Cheng Y, Xie Z, Zang L, Mu Y, Han W. Bone marrow-derived mesenchymal stem cells ameliorate chronic high glucose-induced $\beta$-cell injury through modulation of autophagy. Cell Death Dis. 2015;6(9):e1885.

56. Tamargo-Gómez I, Mariño G. AMPK: regulation of metabolic dynamics in the context of autophagy. Int J Mol Sci. 2018;19(12):3812.

57. Li Y, Chen Y. AMPK and autophagy. Adv Exp Med Biol. 2019;1206:85-108.

58. Miller S, Oleksy A, Perisic O, Williams RL. Finding a fitting shoe for Cinderella: searching for an autophagy inhibitor. Autophagy. 2010;6(6):805-7.

\section{Publisher's Note}

Springer Nature remains neutral with regard to jurisdictional claims in published maps and institutional affiliations. 